\title{
Disease-modifying drugs can reduce disability progression in relapsing multiple sclerosis
}

\begin{abstract}
Maria Pia Amato,, ', Mattia Fonderico,' Emilio Portaccio, ${ }^{3}$ Luisa Pastò,' Lorenzo Razzolini,' Elio Prestipino,' Angelo Bellinvia,' Laura Tudisco,' Roberto Fratangelo,' Giancarlo Comi, ${ }^{4}$ Francesco Patti, ${ }^{5}$ Giovanna De Luca, ${ }^{6}$ Vincenzo Brescia Morra, ${ }^{7}$ Eleonora Cocco, ${ }^{8}$ Carlo Pozzilli, ${ }^{9}$ Patrizia Sola, ${ }^{10}$ Roberto Bergamaschi, ${ }^{1}$ Giuseppe Salemi, ${ }^{12}$ Matilde Inglese, ${ }^{13,14}$ Enrico Millefiorini, ${ }^{15}$ Simonetta Galgani, ${ }^{16}$ Mauro Zaffaroni, ${ }^{17}$ Angelo Ghezzi, ${ }^{17}$ Marco Salvetti, ${ }^{18,19}$ Giacomo Lus, ${ }^{20}$ Ciro Florio, ${ }^{21}$ Rocco Totaro, ${ }^{22}$ Franco Granella, ${ }^{23}$ Marika Vianello, ${ }^{24}$ Maurizia Gatto, ${ }^{25}$ Giancarlo Di Battista, ${ }^{26}$ Umberto Aguglia, ${ }^{27}$ Francesco Ottavio Logullo, ${ }^{28}$ Marta Simone, ${ }^{29}$ Giuseppe Lucisano, ${ }^{30,31}$ (1)Pietro laffaldano ${ }^{31}$ and Maria Trojano ${ }^{31}$ on behalf of the Italian Multiple Sclerosis Register Centers Group
\end{abstract}

See Gaudioso and Naismith (doi:10.1093/brain/awaa295) for a scientific commentary on this article.

1 Department NEUROFARBA, University of Florence, Florence, Italy

2 IRCCS Fondazione Don Carlo Gnocchi, Florence, Italy

3 SOC Neurologia, Ospedale San Giovanni di Dio, AUSL Toscana Centro1, Florence, Italy

4 San Raffaele Hospital - INSPE; Vita-Salute San Raffaele University, Milan, Italy

5 Dipartimento di Scienze Mediche e Chirurgiche e Tecnologie Avanzate, GF Ingrassia, Sez. Neuroscienze, Centro Sclerosi Multipla, University of Catania, Catania, Sicily, Italy

6 Centro Sclerosi Multipla, Clinica Neurologica, Policlinico SS Annunziata, Università 'G. d'Annunzio', Chieti-Pescara, Italy

Received February 15, 2020. Revised May 30, 2020. Accepted June 29, 2020. Advance access publication September 16,2020 (c) The Author(s) (2020). Published by Oxford University Press on behalf of the Guarantors of Brain. All rights reserved.

For permissions, please email: journals.permissions@oup.com 
7 Department of Neuroscience, Reproductive and Odontostomatological Sciences, Federico II University, Napoli, Italy

8 Centro Sclerosi Multipla, ASSL Cagliari (ATS Sardegna); Dipartimento di Scienze Mediche e Sanità Pubblica, University of Cagliari, Cagliari, Italy

9 Multiple Sclerosis Center, S. Andrea Hospital, Dept. of Human Neuroscience, Sapienza University, Rome, Italy

10 Centro Malattie Demielinizzanti - Dipartimento di Neuroscienze, Azienda Ospedaliero-Universitaria/OCSAE, UO Neurologia, University of Modena and Reggio Emilia, Modena, Italy

11 IRCCS Mondino Foundation, Pavia, Italy

12 Department of Biomedicine, Neuroscience and Advanced Diagnostics, University of Palermo, Palermo, Sicily, Italy

13 Department of Neurosciences, Rehabilitation, Ophthalmology, Genetics, Maternal and Child Health (DINOGMI), University of Genoa, Genoa, Italy

14 Ospedale Policlinico San Martino, IRCCS, Genoa, Italy

15 Multiple Sclerosis Center, Policlinico Umberto I, Sapienza University, Rome, Italy

16 multiple sclerosis Centre, Department of Neurosciences, S. Camillo - Forlanini Hospital, Rome, Italy

17 ASST della Valle Olona, Multiple Sclerosis Center, S. Antonio Abate Hospital of Gallarate, Gallarate, Italy

18 Department of Neuroscience, Mental Health and Sensory Organs, Faculty of Medicine and Psychology, Centre for Experimental Neurological Therapies, S. Andrea Hospital/Sapienza University, Rome, Italy

19 IRCCS Istituto Neurologico Mediterraneo (INM) Neuromed, Rome, Italy

20 Università della Campania Luigi Vanvitelli, Naples, Italy

21 Multiple Sclerosis Center, Cardarelli Hospital, Naples, Italy

22 Demyelinating Diseases Center, Department of Neurology, San Salvatore Hospital, L'Aquila, Italy

23 Unit of Neurosciences, Department of Medicine and Surgery, University of Parma, Italy

24 Centro Sclerosi Multipla - Ospedale Regionale 'Ca' Foncello', Neurology Unit, Treviso, Italy

25 Ospedale Generale Regionale 'F. Miulli', Neurology Unit, Acquaviva delle Fonti (BA), Italy

26 Centro Sclerosi Multipla, ASL Roma 1, PO S. Filippo Neri, Rome, Italy

27 Department of Medical and Surgical Sciences, Magna Graecia University of Catanzaro, Neurology Unit, Catanzaro, Italy

28 Centro Sclerosi Multipla-UOC Neurologia-Ospedale di Macerata, Macerata, Italy

29 Child Neuropsychiatric Unit, Department of Biomedical Sciences and Human Oncology, University 'Aldo Moro’ of Bari, Policlinico Piazza G. Cesare, 11, 70121, Bari, Italy

30 Center for Outcomes Research and Clinical Epidemiology, Pescara, Italy

31 Department of Basic Medical Sciences, Neurosciences and Sense Organs, University of Bari ‘Aldo Moro’ Policlinico, Bari, Italy

Correspondence to: Mattia Fonderico

Department NEUROFARBA, University of Florence, Florence, Italy

E-mail: mattia.fonderico1991@gmail.com

Keywords: demyelination; multiple sclerosis epidemiology; clinical trials; neuroinflammation; clinically isolated syndrome

Abbreviations: AOMS = adult-onset multiple sclerosis; CDW = confirmed disability worsening; DMT = disease-modifying therapy; EDSS = Expanded Disability Status Scale; LOMS = late-onset multiple sclerosis; POMS = paediatric-onset multiple sclerosis;

$\mathrm{RCT}=$ randomized clinical trial; RRMS = relapsing-remitting multiple sclerosis

\section{Introduction}

Multiple sclerosis is an inflammatory demyelinating disease of the CNS that is characterized by inflammation, demyelination, and degenerative changes. Most individuals are diagnosed with multiple sclerosis at age 20-40 years (Finlayson, 2004). Paediatric-onset multiple sclerosis (POMS), before the age of 18 , represents $3-10 \%$ of the whole multiple sclerosis population (Iaffaldano et al., 2017), while late-onset multiple sclerosis (LOMS), after the age of 50, now accounts for $3-5 \%$ of all multiple sclerosis diagnosis (Vaughn et al., 2019). Age at onset plays an important prognostic role, not fully understood, and may impact disease course and treatment response. Clinic (Tutuncu et al., 2013) and population (Confavreux and Vukusic, 2006a; Kremenchutzky et al., 2006) based studies suggested that the onset of the progressive phase and time to Expanded Disability Status Scale (EDSS) milestones is an age-dependent phenomenon, independent of the initial course of multiple sclerosis. Nevertheless, predicting disability accumulation only on chronological age would be an oversimplification. In both POMS and adult-onset multiple sclerosis (AOMS), the strongest predictor for reaching the EDSS milestones is age at clinical onset: the earlier the onset of disease, the younger the age at which the main disability milestones are reached (Confavreux and Vukusic, 2006b; Renoux et al., 2007). Moreover, considering disease progression solely as an agedependent phenomenon would hide the high variability in progression rate observed among individuals with multiple sclerosis (Tremlett et al., 2006).

Additionally, age at onset influences the response to disease-modifying therapy (DMT). A recent meta-analysis of the main randomized clinical trials (RCTs) demonstrated that the efficacy of DMTs on disability worsening has an inverse correlation with increasing age (Weideman et al., 2017). These clinical results seem to be in line with the 
progressive weakening of both adaptive and innate immune system called immunosenescence (Musella et al., 2018). This model, however, was based on RCTs that generally excluded patients younger than 18 or older than 50 years. As the prevalence of LOMS is increasing (Vaughn et al., 2019), data regarding DMT effectiveness in this group of patients are warranted. This is even more relevant due to comorbidity and possibly higher risks of treatment-related adverse events in this age group (Schweitzer et al., 2019). As for the paediatric counterpart, clinical trials in POMS subjects are extremely limited, also due to ethical considerations on the use of placebo owing to highly active disease in this population (Waubant et al., 2019). Although most of the DMTs are not licensed for POMS, their off-label prescription is increasing in this subpopulation (Iaffaldano et al., 2017). Therefore, benefit to risk balance and treatment decisionmaking in these extreme age populations present unique age- and disease-related challenges.

As a sizeable proportion of paediatric and older patients are treated in the real-world setting, registry-based cohort studies represent a major source of data to elucidate the above issues.

The research question, addressed in this multicentre study based on the Italian multiple sclerosis register, was whether and how treatment response differ in three cohorts of patients with relapsing-remitting multiple sclerosis (RRMS) defined by age at onset: POMS ( $\leqslant 18$ years), AOMS (18-49 years) and LOMS ( $\geqslant 50$ years).

\section{Patients and methods}

\section{Ethics statement}

The Italian iMedWeb network was approved by the Policlinico of Bari Ethics Committee and by the local ethics committees in all participating centres. Written informed consent was obtained from all enrolled patients, or in the case of POMS from their parents, in accordance with the Declaration of Helsinki.

\section{Study design}

We conducted a multicentre, observational, retrospective cohort study based on prospectively acquired clinical data. The aim was to evaluate how the effectiveness of DMTs can vary in three subgroups of RRMS patients defined by their age at onset. Anonymized clinical records of patients with a first demyelinating event were extracted from the Italian multiple sclerosis register (Trojano et al., 2019) in November 2018. Inclusion criteria were: patients with a first neurological evaluation within 3 years from the first demyelinating event; a minimum of three visits with EDSS evaluation; a minimum of 5 years follow-up. We excluded patients with a primary progressive course and those enrolled in RCTs. Multiple sclerosis duration was calculated from the first demyelinating event. The follow-up time was defined as the time between the first and last available EDSS entry.

The Italian multiple sclerosis register protocol requires, for all patients registered, a minimum baseline dataset (Trojano et al.,
2019). The minimum dataset required for this study also comprised clinical course, follow-up visit dates, EDSS scores recorded at each visit, date of all relapses, start and end dates of all DMT commencements and DMT type. Quality assurance through online certification of EDSS competency is required at each participating site.

RRMS patients meeting the eligibility criteria were divided into three subgroups according to their age at the first demyelinating event: POMS ( $\leqslant 18$ years), AOMS (19-49 years) and LOMS ( $\geqslant 50$ years).

We considered the following outcomes: 12-month confirmed disability worsening (CDW) and EDSS 4.0. As an exploratory outcome, we also considered the time to EDSS 6.0. A minimum of three visits per patient over a minimum period of 12 months, with complete EDSS assessment, was required to assess the first 12-month CDW.

CDW events were defined as $\geqslant 12$-month confirmed increase of: $\geqslant 1.0$ point for those with a baseline EDSS score between 1.0 and 5.5 , inclusive; and $\geqslant 1.5$ points for those with a baseline EDSS score of 0 . Irreversible assignment of EDSS score 4.0 or 6.0 was defined as reaching of EDSS score 4.0 or 6.0 with all subsequent EDSS scores being either equal to these scores, or greater. EDSS recorded within 30 days from a clinical relapse were excluded to avoid artificial increase of EDSS score changes over time. The Italian MS Register protocol stipulates a required biannual update of the minimum dataset, but patients with less frequent visits were not excluded from the analyses. The baseline of the study was the first visit. For each subgroup, we evaluated as possible prognostic predictors sex, symptom at onset (multifocal/unifocal), number of relapses, number of EDSS evaluations and treatment exposure.

\section{Statistical analysis}

The baseline and follow-up characteristics were expressed as mean and standard deviation (SD) or frequency and percentage for continuous and categorical covariates, respectively. Categorical and continuous variables were compared by using $\chi^{2}$ statistic and Kruskal-Wallis test, respectively. Non-parametric tests were most conservative.

Predictors of first 12-month CDW and EDSS 4.0 were assessed using multivariable Cox proportional hazard regressions. The date of the first visit with full EDSS evaluation was used as time origin of the model to mitigate a possible immortal time bias. In the absence of a worsening or reaching EDSS 4.0, data were censored at the latest EDSS available. Results of Cox regression models were expressed as hazard ratio (HR) and $95 \%$ confidence interval (CI) of reaching the outcomes. We performed different models for each subgroup of patient (POMS, AOMS and LOMS). The multivariable modelling analyses were adjusted for the following covariates: sex (female versus male), symptom at onset (multifocal versus unifocal), number of relapses, percentage of time spent before the outcome (in quartiles) and EDSS score at first visit. As the visit frequency was different across the three cohorts, we further adjusted all the multivariate models for the number of EDSS evaluations. For DMT exposure, we considered the total time a patient spent on treatment, including any switches and gaps in treatment. During the survival time, we did not consider gaps $\leqslant 3$ months as a therapy interruption. As the percentage spent on DMT before the outcome was not normally distributed, a statistical stratification in 
four groups, of equal size (quartiles), was used. The fourth quartile (Q4), including patients who were above the 75th percentile, was regarded as the reference class in the Cox models. In the present study, 'never treated' was the patient group that did not receive any DMT treatment before the first disability worsening or EDSS 4.0 attainment. The exposure time was censored at the reaching of the outcome or at the last visit if a worsening event had not yet occurred. A sensitivity analysis was carried out by including patients registered after 2000 .

All statistical analyses were performed with $\mathrm{R}$ version 3.2.0 and $P$-value $<0.05$ was considered statistically significant.

\section{Data availability}

Anonymized data, not published in the article, will be shared on reasonable request from a qualified investigator.

\section{Results}

\section{Baseline and follow-up characteristics}

The data extraction was completed in November 2018. We had access to 55669 register patients from 77 Italian centres. By applying inclusion and exclusion criteria, we identified 646 POMS, 8473 AOMS and 382 LOMS patients at the first demyelinating event (Fig. 1). The baseline and follow-up characteristics of the three cohorts are described in Tables 1 and 2 .

There was a comparable female prevalence in all three groups. Spinal cord symptoms at onset were more frequent in LOMS $(P<0.001)$, whereas optic neuritis was more represented in the two younger cohorts. LOMS patients had, on average, a higher mean EDSS baseline score compared with the other two cohorts $(P<0.001)$.

As for the first treatment (Table 1), we distinguished between moderately effective DMTs (IFN $\beta 1 \mathrm{a}$, IFN $\beta 1 \mathrm{~b}$, glatiramer, dimethyl fumarate, teriflunomide and azathioprine) and highly effective DMTs (monoclonal antibodies, mitoxantrone, cladribine and fingolimod) (Rotstein and Montalban, 2019).

The vast majority of patients in the three groups received a moderately effective DMT as first treatment. Compared with the other two groups, a higher proportion of LOMS subjects $(21.8 \%)$ were never exposed to a DMT (Table 1).

The mean follow-up time was $\sim 12$ years for POMS, 11 years for AOMS and 9 years for LOMS patients $(P<0.0001)$. Mean disease duration was longer in POMS (13 years) compared with AOMS (12 years) and LOMS (10 years). The first EDSS evaluation was made, on average, within 13 months from disease onset in all the three cohorts $(P=0.28)$. The mean number of EDSS evaluations was higher in the two younger cohorts $(P<0.0001)$; moreover, LOMS patients exhibited lower annualized EDSS evaluations compared with the two younger cohorts $(P=0.005)$.

All three cohorts received the first DMT, on average, within 3 years from symptom onset, without any significant difference $(P=0.568)$. LOMS patients spent a lower percentage of follow-up time under a DMT than the other two cohorts (5.8 versus 6.8 and 7.2 years of AOMS and POMS patients, respectively $P=0.003)$. During the followup period, the mean number of relapses was higher in POMS (6.8) and AOMS (5.3) compared with LOMS (3.7) subjects, $P<0.0001$.

The frequency of switch from moderate to highly effective DMTs was significantly higher in the POMS and AOMS compared with the LOMS cohort, $P<0.0001$ (Table 2).

In terms of outcome, the LOMS cohort experienced a higher rate of 12-month CDW and accrual of EDSS 4.0 than the two younger cohorts $(P<0.0001)$, although the number of EDSS evaluations was lower (Table 2).

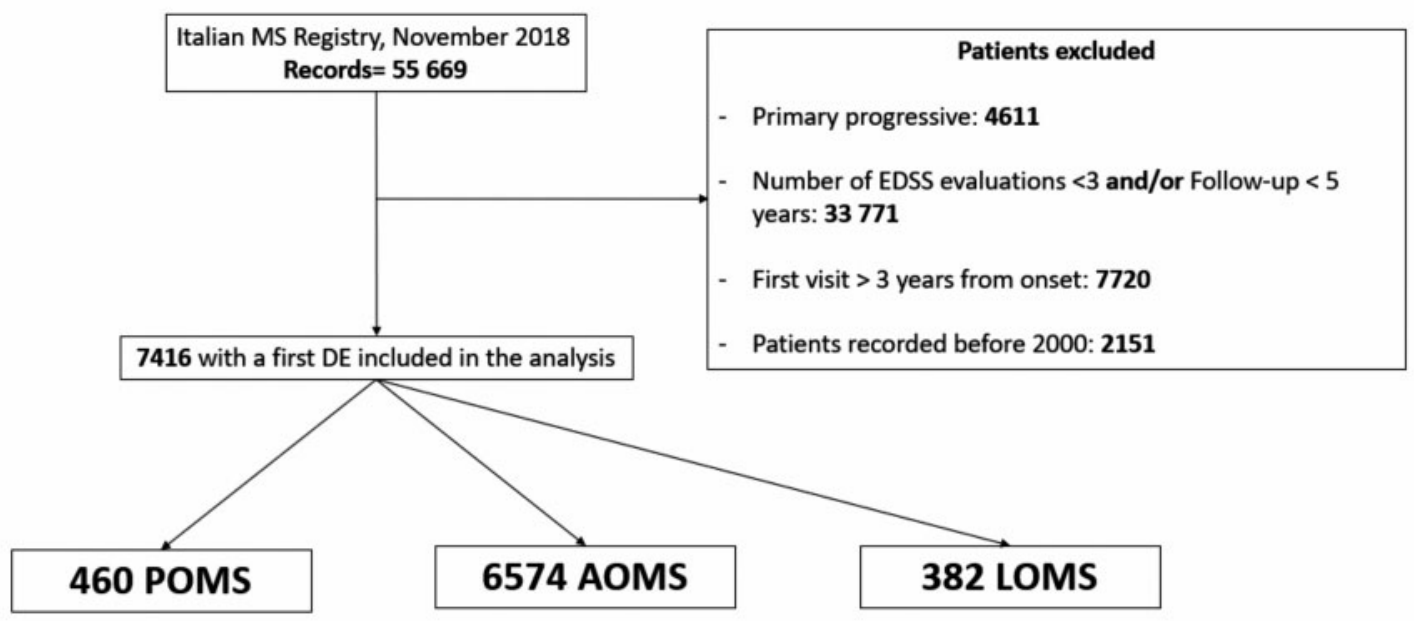

Figure I Flow-chart showing patients selection. DE = demyelinating event. 
Table I Baseline characteristics of the three cohorts defined by age at onset

\begin{tabular}{|c|c|c|c|c|}
\hline Total, $n=9567$ & $\begin{array}{l}\text { Paediatric-onset } \\
n=646\end{array}$ & $\begin{array}{l}\text { Adult-onset } \\
n=8473\end{array}$ & $\begin{array}{l}\text { Late-onset } \\
n=448\end{array}$ & $P$-value \\
\hline Age at onset, mean \pm SD & $15.48 \pm 2.43$ & $31.21 \pm 7.91$ & $54.29 \pm 9.20$ & $<0.0001$ \\
\hline Sex, female $n(\%)$ & $448(69.35)$ & $5704(67.32)$ & $299(66.74)$ & $0.54 I$ \\
\hline \multicolumn{5}{|l|}{ CIS topography $n(\%)$} \\
\hline Optic neuritis & $156(24.15)$ & $1818(21.48)$ & $67(15.02)$ & $<0.0001$ \\
\hline Brainstem symptoms & $140(21.67)$ & $1646(19.44)$ & $90(20.18)$ & \\
\hline Spinal symptoms & $82(12.69)$ & $1659(19.60)$ & $113(25.34)$ & \\
\hline Supratentorial symptoms & $170(26.32)$ & $2167(25.60)$ & $124(27.80)$ & \\
\hline Combination of symptoms & $98(15.17)$ & $1175(13.88)$ & $52(11.66)$ & \\
\hline First EDSS score, mean \pm SD & $1.52 \pm 1.12$ & $1.68 \pm 1.21$ & $2.39 \pm 1.51$ & $<0.0001$ \\
\hline \multicolumn{5}{|l|}{ Treatment $n(\%)$} \\
\hline First DMT, moderate efficacy & $583(90.25)$ & $7378(87.01)$ & $336(75.0)$ & 0.167 \\
\hline First DMT, high efficacy & $34(5.26)$ & $336(4.36)$ & $14(4.00)$ & \\
\hline Never treated & $29(4.48)$ & $759(8.96)$ & $98(21.8)$ & \\
\hline Percentage of follow-up spent on DMT & $43.52 \pm 27.04$ & $39.99 \pm 27.47$ & $36.52 \pm 27.71$ & 0.003 \\
\hline Time to first DMT, days & $959.46 \pm|34| .97$ & $880.46 \pm|| 32.5 \mid$ & $835.28 \pm 919.36$ & 0.568 \\
\hline
\end{tabular}

Moderate efficacy DMTs comprise IFN $\beta$ Ia, IFN $\beta$ Ib, glatiramer, dimethyl fumarate, teriflunomide and azathioprine. High efficacy DMTs comprise monoclonal antibodies, mitoxantrone, cladribine and fingolimod. CIS = clinically isolated syndrome.

Table 2 Follow-up characteristics of the three cohorts defined by age at onset

\begin{tabular}{|c|c|c|c|c|}
\hline Total $n=9567$ & $\begin{array}{l}\text { Paediatric-onset MS } \\
n=646\end{array}$ & $\begin{array}{l}\text { Adult-onset MS } \\
n=8473\end{array}$ & $\begin{array}{l}\text { Late-onset MS } \\
n=448\end{array}$ & $P$-value \\
\hline Follow-up, years, mean \pm SD & $12.18 \pm 6.09$ & $11.30 \pm 5.79$ & $9.47 \pm 3.60$ & $<0.0001$ \\
\hline Disease duration, years, mean $\pm S D$ & $|3.18 \pm 5.8|$ & $12.27 \pm 5.85$ & $10.03 \pm 9.83$ & $<0.0001$ \\
\hline Time to first EDSS evaluation, months, mean \pm SD & $13.01 \pm 10.33$ & $12.46 \pm 10.12$ & $13.02 \pm 10.35$ & 0.28 \\
\hline Number of EDSS evaluations per patient, mean \pm SD & $21.73 \pm 13.30$ & $19.72 \pm 13.24$ & $|5.8| \pm 10.07$ & $<0.0001$ \\
\hline Mean annualized EDSS evaluation, mean \pm SD & $1.94 \pm 1.08$ & $1.87 \pm 1.12$ & $1.76 \pm 1.00$ & 0.005 \\
\hline Time to first DMT, days, mean \pm SD & $959.46 \pm 1341.97$ & $880.46 \pm|| 132.5 \mid$ & $835.28 \pm 919.36$ & 0.5685 \\
\hline Time spent on DMT, years & $7.18 \pm 4.14$ & $6.81 \pm 4.77$ & $5.83 \pm 3.79$ & $<0.0001$ \\
\hline \multicolumn{5}{|l|}{ Quartiles of time spent on DMT, $n(\%)(n=6353)$} \\
\hline QI (I-19.6\%) & II 15 (22.07) & $1416(25.17)$ & $58(28.16)$ & 0.099 \\
\hline Q2 (19.7-40.4\%) & $113(21.69)$ & $1420(25.24)$ & $55(26.70)$ & \\
\hline Q3 (40.5-65.5\%) & $149(28.60)$ & $1393(24.76)$ & $46(22.33)$ & \\
\hline Q4 (>65.5\%) & $144(27.64)$ & $1397(24.83)$ & $47(22.82)$ & \\
\hline Number of relapses, mean \pm SD & $6.80 \pm 5.08$ & $5.26 \pm 3.94$ & $3.72 \pm 2.39$ & $<0.0001$ \\
\hline Mean annualized assessment rate of relapses & $0.59 \pm 0.37$ & $0.50 \pm 0.34$ & $0.42 \pm 0.27$ & $<0.0001$ \\
\hline Patients who changed from moderate to high efficacy DMT, $n(\%)$ & $253(38.57)$ & $1819(21.47)$ & $42(9.37)$ & $<0.0001$ \\
\hline \multicolumn{5}{|l|}{ Outcome, $n(\%)$} \\
\hline 12 months CDW & $128(19.81)$ & $2078(24.52)$ & $150(33.48)$ & $<0.0001$ \\
\hline EDSS $4.0^{\mathrm{a}}$ & $67(10.81)$ & $1496(18.66)$ & $130(34.57)$ & $<0.0001$ \\
\hline
\end{tabular}

Moderate efficacy DMTs comprise IFN $\beta$ I a, IFN $\beta$ I b, glatiramer, dimethyl fumarate, teriflunomide and azathioprine. High efficacy DMTs comprise monoclonal antibodies, mitoxantrone, cladribine and fingolimod.

${ }^{\mathrm{a}}$ For the outcome EDSS 4.0 the patients with a first EDSS $\geqslant 4$ were excluded.

\section{Treatment effect on I 2-month confirmed disability worsening and EDSS 4.0}

Table 3 shows the results of the adjusted multivariate Cox regression model for the outcome 12-month CDW, whereas Table 4 shows the results for the outcome EDSS 4.0. To mitigate for possible secular trend bias, the same models were run as sensitivity analyses by including only patients registered after 2000 (Tables 5 and 6). The results were adjusted for sex, type of onset (unifocal versus multifocal), number of relapses and of EDSS evaluations and, for the EDSS 4.0 outcome, for baseline EDSS score. We evaluated the treatment effect with a time-dependent approach considering the total time a patient spent on treatment, including any switches and gaps. We further divided exposure time into quartiles. The fourth quartile (Q4) was the quartile of patients who were above the 75 th percentile and has been 
Table 3 Multivariate Cox model for the first I2-month confirmed disability worsening in the overall sample $(n=9567)$

\begin{tabular}{|c|c|c|c|c|}
\hline Overall sample, $n=9567$ & & $\begin{array}{l}\text { Paediatric-onset MS } \\
n=646\end{array}$ & $\begin{array}{l}\text { Adult-onset MS } \\
n=8473\end{array}$ & $\begin{array}{l}\text { Late-onset MS } \\
n=448\end{array}$ \\
\hline Number of events, $n$ (\%) & & $128(19.8 \%)$ & $2078(24.5 \%)$ & $150(33.4)$ \\
\hline Variable & Reference class & aHR $(95 \% \mathrm{Cl})$ & aHR $(95 \% \mathrm{Cl})$ & aHR $(95 \% \mathrm{Cl})$ \\
\hline Sex, female & Male & $1.15(0.78-1.70)$ & $1.04(0.95-1.14)$ & $0.74(0.53-1.04)$ \\
\hline Symptom at onset, multifocal & Unifocal & $1.06(0.67-1.68)$ & $0.87(0.78-0.98)^{*}$ & $1.13(0.69-1.85)$ \\
\hline Number of relapses & - & $1.17(1.14-1.20)^{* *}$ & $1.17(1.17-1.18)^{* *}$ & $1.40(1.32-1.48)^{* * *}$ \\
\hline Number of EDSS evaluations & - & $0.97(0.95-0.98)^{* *}$ & $0.98(0.98-0.99)^{* * *}$ & $0.98(0.96-1.00)^{*}$ \\
\hline DMT exposure ${ }^{a}$ & $\mathrm{Q} 4^{\mathrm{b}}$ & & & \\
\hline Never treated & & $7.98(3.55-17.95)^{* *}$ & $6.27(4.92-7.98)^{* *}$ & $1.95(0.94-4.06)$ \\
\hline QI & & $3.69(1.61-8.48)^{*}$ & $4.46(3.47-5.73)^{* * *}$ & $1.10(0.47-2.54)$ \\
\hline Q2 & & $3.59(1.53-8.44)^{*}$ & $3.07(2.37-3.97)^{* * *}$ & $1.40(0.61-3.25)$ \\
\hline Q3 & & $2.94(1.22-7.06)^{*}$ & $2.30(1.76-3.02)^{\text {*** }}$ & $0.85(0.32-2.28)$ \\
\hline
\end{tabular}

aHR = adjusted hazard ratio; MS = multiple sclerosis.

${ }^{a}$ For DMT exposure, we considered the total time a patient spent on treatment, including any switches and/or gaps.

bWe divided exposure time into quartiles. The fourth quartile (Q4) is the quartile of patients who were above the 75th percentile and has been regarded as the reference class in the Cox models. 'Never treated' were the patients who did not receive any DMT before the first 12 months disability worsening or EDSS 4.0 . The exposure time was censored at the reaching of the outcome or most recent visit if a worsening event had not yet occurred.

$* P$-value $<0.05 ; * * P$-value $<0.001$.

Table 4 Multivariate Cox model for the risk of reaching EDSS 4 in the overall sample $(n=90 I 2)$

\begin{tabular}{|c|c|c|c|c|}
\hline Overall sample $(n=9012)$ & & $\begin{array}{l}\text { Paediatric-onset MS } \\
n=620\end{array}$ & $\begin{array}{l}\text { Adult-onset MS } \\
n=8016\end{array}$ & $\begin{array}{l}\text { Late-onset MS } \\
n=374\end{array}$ \\
\hline Number of events, $n$ (\%) & & $67(10.81)$ & $1496(18.66)$ & $130(34.57)$ \\
\hline Variable & Reference class & aHR $(95 \% \mathrm{Cl})$ & aHR $(95 \% \mathrm{Cl})$ & $\operatorname{aHR}(95 \% \mathrm{Cl})$ \\
\hline Sex, female & Male & $0.91(0.53-1.59)$ & $0.97(0.87-1.08)$ & $0.42(0.29-0.60)^{* *}$ \\
\hline Symptom at onset, multifocal & Unifocal & $0.92(0.49-1.7 \mathrm{I})$ & $1.03(0.90-1.19)$ & $1.52(0.87-2.64)$ \\
\hline First EDSS & - & $1.48(1.12-1.97)^{*}$ & $1.76(1.66-1.86)^{* *}$ & $1.89(1.55-2.29)^{* * *}$ \\
\hline Number of relapses & - & $1.15(1.12-1.18)^{* *}$ & $1.17(1.16-1.17)^{* *}$ & $1.49(1.39-1.59)^{* *}$ \\
\hline Number of EDSS evaluations & - & $0.99(0.97-1.01)$ & $1.00(1.00-1.01)^{*}$ & $1.00(0.99-1.02)$ \\
\hline DMT exposure ${ }^{a}$ & $\mathrm{Q} 4^{\mathrm{b}}$ & & & \\
\hline Never treated & & $7.06(2.05-24.34)^{*}$ & $10.34(7.42-14.40)^{* * *}$ & $9.36(1.30-67.67)^{*}$ \\
\hline QI & & $4.76(1.35-16.76)^{*}$ & $6.67(4.74-9.38)^{* * k}$ & $9.21(1.22-69.5 \mathrm{I})^{*}$ \\
\hline Q2 & & $3.86(1.07-13.97)^{*}$ & $4.44(3.13-6.30)^{* * *}$ & $3.80(0.48-29.8 I)$ \\
\hline Q3 & & $3.17(0.87-11.58)$ & $3.44(2.39-4.95)^{* *}$ & $2.95(0.35-24.64)$ \\
\hline
\end{tabular}

aHR = adjusted hazard ratio; $M S=$ multiple sclerosis.

${ }^{\mathrm{a}}$ For DMT exposure, we considered the total time a patient spent on treatment, including any switches and/or gaps.

bWe divided exposure time into quartiles. The fourth quartile (Q4) is the quartile of patients who were above the 75th percentile and has been regarded as the reference class in the Cox models. 'Never treated' were the patients who did not receive any DMT before the first I2-month disability worsening or EDSS 4.0. The exposure time was censored at the reaching of the outcome or most recent visit if a worsening event had not yet occurred.

$* P$-value $<0.05 ; * * P$-value $<0.001$.

regarded as the reference class in the Cox models. In the present study, patients who did not receive any DMT before reaching the two clinical outcomes represented the 'never treated' class. The exposure time was censored at the reaching of the outcome or the last visit if a worsening event had not occurred yet.

Female sex was a protective factor for LOMS patients decreasing the risk of reaching EDSS 4.0 of $58 \%$ [adjusted HR $(\mathrm{aHR})=0.42,95 \%$ CI $0.29-0.60, \quad P<0.001]$. Sensitivity analyses confirmed the same protective role with a similar magnitude.

A higher number of relapses was associated with an increased risk of both 12-month CDW and EDSS 4.0 in all the three cohorts (Tables 3 and 4). These results were also confirmed in the sensitivity analyses, with an adjusted HR for 12-month-CDW and EDSS 4.0 ranging from 1.15 to 2.11 (Tables 5 and 6).

As for type of onset (multifocal versus unifocal), a multifocal onset showed a weak protective effect for a first 12month CDW in AOMS $(\mathrm{aHR}=0.87,95 \% \mathrm{CI}=0.78-0.98$, $P<0.005)$ but did not significantly influence the risk of reaching the EDSS 4.

The most significant predictive factor of 12 -month CDW was DMT treatment, which was especially evident in the two younger cohorts. In POMS and AOMS, increasing the DMT exposure resulted in a stepwise reduction of $\sim 2$ points 
Table 5 Multivariate Cox model for the first I2-month confirmed disability worsening performed in patients with disease onset after $2000(n=7416)$

\begin{tabular}{|c|c|c|c|c|}
\hline Overall sample $(n=7416)$ & & $\begin{array}{l}\text { Paediatric-onset MS } \\
n=460\end{array}$ & $\begin{array}{l}\text { Adult-onset MS } \\
n=6574\end{array}$ & $\begin{array}{l}\text { Late-onset MS } \\
n=382\end{array}$ \\
\hline Number of events, $n$ (\%) & & $52(11.30)$ & I $205(\mid 8.33)$ & $115(30.10)$ \\
\hline Variable & Reference class & aHR $(95 \% \mathrm{Cl})$ & aHR $(95 \% \mathrm{Cl})$ & aHR $(95 \% \mathrm{Cl})$ \\
\hline Sex, female & Male & $0.64(0.33-1.25)$ & $1.02(0.90-1.15)$ & $0.74(0.5 \mathrm{I}-1.09)$ \\
\hline Symptom at onset, multifocal & Unifocal & $1.06(0.44-2.55)$ & $0.95(0.8 I-1.1 I)$ & $1.54(0.86-2.77)$ \\
\hline Number of EDSS evaluations & - & $0.95(0.93-0.98)^{*}$ & $0.97(0.97-0.98)^{* * k}$ & $0.99(0.97-1.01)$ \\
\hline Number of relapses & - & $1.41(1.33-1.49)^{* * *}$ & $1.37(1.36-1.39)^{* *}$ & $1.40(1.31-1.49)^{* *}$ \\
\hline DMT exposure ${ }^{\mathrm{a}}$ & $\mathrm{Q} 4^{\mathrm{b}}$ & & & \\
\hline Never treated & & $9.71(2.72-34.61)^{* * *}$ & $6.96(5.14-9.43)^{* k}$ & I.82 (0.83-3.99) \\
\hline QI & & $6.88(1.92-24.72)^{*}$ & $4.21(3.07-5.76)^{* 6 /}$ & $0.8 \mathrm{I}(0.32-2.03)$ \\
\hline Q2 & & $2.25(0.60-8.50)$ & $4.17(3.03-5.73)^{* 6 k}$ & $1.61(0.68-3.86)$ \\
\hline Q3 & & $2.34(0.6 \mathrm{I}-8.93)$ & $2.45(1.75-3.43)^{* * *}$ & $0.43(0.11-1.66)$ \\
\hline
\end{tabular}

aHR = adjusted hazard ratio; MS = multiple sclerosis.

${ }^{a}$ For DMT exposure, we considered the total time a patient spent on treatment, including any switches and/or gaps.

${ }^{b}$ We divided exposure time into quartiles. The fourth quartile (Q4) is the quartile of patients who were above the 75th percentile and has been regarded as the reference class in the Cox models. 'Never treated' were the patients who did not receive any DMT before the first I2-month disability worsening or EDSS 4.0. The exposure time was censored at the reaching of the outcome or most recent visit if a worsening event had not yet occurred.

$* P$-value $<0.05 ; * * P$-value $<0.001$.

Table 6 Multivariate Cox model for the risk of reaching EDSS 4.0 in patients with disease onset after 2000 $(n=7006)$

\begin{tabular}{|c|c|c|c|c|}
\hline Overall sample $(n=7006)$ & & $\begin{array}{l}\text { Paediatric-onset MS } \\
n=444\end{array}$ & $\begin{array}{l}\text { Adult-onset MS } \\
n=6234\end{array}$ & $\begin{array}{l}\text { Late-onset MS } \\
n=328\end{array}$ \\
\hline Number of events, $n$ (\%) & & $25(5.63)$ & $857(13.75)$ & I04 (3I.7I) \\
\hline Variable & Reference class & aHR (95\%Cl) & aHR (95\%Cl) & aHR $(95 \% \mathrm{Cl})$ \\
\hline Sex, female & Male & $0.82(0.30-2.24)$ & $0.80(0.69-0.92)^{*}$ & $0.44(0.29-0.67)^{* *}$ \\
\hline Symptom at onset, multifocal & Unifocal & $0.74(0.20-2.72)$ & $0.83(0.69-0.99)^{*}$ & $2.04(1.07-3.90)^{*}$ \\
\hline Number of EDSS evaluations & - & $0.96(0.91-1.01)$ & $1.01(1.01-1.02)^{* *}$ & $1.01(0.99-1.03)$ \\
\hline Number of relapses & - & $1.60(1.42-1.80)^{* * *}$ & $1.31(1.29-1.32)^{* * *}$ & $1.48(1.38-1.59)^{* *}$ \\
\hline First EDSS & - & $2.16(1.24-3.78)^{*}$ & $1.91(1.76-2.06)^{* *}$ & $\mathrm{I} .87(\mathrm{I} .5 \mathrm{I}-2.32)^{* *}$ \\
\hline DMT exposure ${ }^{a}$ & $\mathrm{Q} 4^{\mathrm{b}}$ & & & \\
\hline Never treated & & $30.39(5.14-179.76)^{* *}$ & $8.97(6.13-13.11)^{* *}$ & $7.75(1.07-56.38)^{*}$ \\
\hline QI & & $7.19(1.17-44.14)^{*}$ & $6.69(4.54-9.87)^{* * *}$ & $8.04(1.07-60.42)$ \\
\hline Q2 & & $6.46(1.11-37.44)^{*}$ & $3.96(2.65-5.92)^{* *}$ & $3.38(0.42-27.50)$ \\
\hline Q3 & & $2.98(0.45-19.83)$ & $1.33(0.85-2.08)^{* *}$ & $2.73(0.33-22.91)$ \\
\hline
\end{tabular}

aHR = adjusted hazard ratio; $M S=$ multiple sclerosis

${ }^{\text {a}}$ For DMT exposure, we considered the total time a patient spent on treatment, including any switches and/or gaps.

bWe divided exposure time into quartiles. The fourth quartile (Q4) is the quartile of patients who were above the 75th percentile and has been regarded as the reference class in the Cox models. 'Never treated' were the patients who did not receive any DMT before the first I2-month disability worsening or EDSS 4.0. The exposure time was censored at the reaching of the outcome or most recent visit if a worsening event had not yet occurred.

$* P$-value $<0.05 ; * *$-value $<0.001$.

in the rate of 12 -month CDW. In the same cohorts, adjusting for baseline EDSS and number of EDSS evaluations, we found similar results considering the risk of reaching EDSS 4.0. Furthermore, the sensitivity models confirmed that the protective role of treatment in POMS and AOMS is related to the cumulative time spent under treatment, seemingly in a time-dependent manner.

As for the LOMS cohort, there was a trend towards a DMT treatment-related lower risk of 12-month CDW. We found that never treated, and the first quartile classes were at higher risk of EDSS 4.0 (Table 4). The sensitivity analyses performed on patients registered after 2000 confirmed the results of the primary model (Tables 5 and 6).
As an exploratory analysis, we investigated the risk of reaching EDSS 6.0. In the AOMS cohort, treatment exposure confirmed its protective role in all the four quartiles. In the LOMS cohort, never treated patients confirmed to be at higher risk of reaching this disability milestone. In the POMS cohort, where only $4.8 \%$ of the subjects reached EDSS 6.0, the results were not statistically significant (Supplementary Table 1).

\section{Discussion}

In recent years, various modern DMTs for multiple sclerosis have become available after demonstrating efficacy in 
clinical trials. However, after evaluation and approval by regulatory agencies, in the real-world setting, these drugs are prescribed in patient populations that differ from those included in pivotal studies. Age can represent a key factor that could change the benefit-risk balance and the therapeutic choice, as it may associate with different efficacy and risk of adverse events of DMTs. Therapeutic decision-making can be particularly challenging in paediatric and older patients who are usually excluded from RCTs. As observational data from registry studies are, therefore, of critical relevance to address issues that are otherwise difficult or impossible to study (Trojano et al., 2017). In this multicentre registry Italian study, we aimed to elucidate how different age at onset can influence the prognostic outcomes and effectiveness of DMTs in preventing disability worsening.

Our real-world data covered a mean follow-up period of $\sim 10$ years in nearly 9500 patients. Prior works suggested that a 12-month confirmation period of disability worsening is a robust outcome measure as it reliably captures irreversible disability accrual (Trojano et al., 2018). Furthermore, EDSS 4.0 is a meaningful clinical milestone as it marks the patient reduced autonomy in ambulation. Moreover, the results of our exploratory analysis of EDSS 6.0 altogether were in line with those of the primary analysis. However, they did not reach the statistical significance in POMS patients, due to the low number of events in this cohort. It is likely that a longer follow-up period is needed to fully investigate harder disability milestones (Tremlett et al., 2006; Scalfari et al., 2013; Cree et al., 2016).

In most of our patients the first treatment was represented by a moderately effective drug. However, during the followup period, the two younger cohorts, with more clinically active disease, exhibited a higher proportion of patients who switched to a highly effective drug.

Overall, our study showed that DMTs can reduce the risk of 12-month CDW and accrual of EDSS 4.0. These findings are consistent with previous observational studies performed with the use of immunomodulatory drugs (Kappos et al., 2009; Jokubaitis et al., 2016; Trojano et al., 2018). In particular, in our AOMS cohort, we found that, in different quartiles of exposure, the risk reduction was related to the cumulative time spent under therapy. This adds evidence that the longer the exposure time, the more likely the protective role against a confirmed disability progression (Trojano et al., 2009; Uitdehaag et al., 2009, 2011; Jokubaitis et al., 2015, 2016), which was also confirmed in sensitivity analyses. These findings are in line with those of Jokubaitis et al. (2015) who found a significant benefit on 12 -month CDW in patients treated for $>50 \%$ of the observation period, versus $<50 \%$ of the time. Notably, we also found very similar results in our large POMS cohort (Alroughani and Boyko, 2018). An international consensus (Chitnis et al., 2012) highlighted the importance for early initiation of DMTs in children and adolescents with multiple sclerosis. POMS patients have, on average, a higher relapse rate, shorter duration between relapses and subsequent accumulation of disability. Although progression may be slower than in adults, moderate-to-severe disability is reached at a younger age (Alroughani and Boyko, 2018). Moreover, although the recovery from relapses seems to be more efficient in POMS because of neuronal plasticity, cognitive impairment is observed in nearly one-third of patients (Amato et al., 2014). To date, however, the only approved DMTs in paediatric multiple sclerosis ( $>10$ years) are interferons, glatiramer and, more recently, fingolimod. The literature describing the effectiveness of DMTs in POMS is limited to observational studies with small sample size (Ghezzi et al., 2016; Alroughani and Boyko, 2018; Waubant et al., 2019). Paediatric clinical trials are challenging due to ethical concerns and difficulties in the recruitment, the study follow-up and site-specific issues (Waubant et al., 2019). In this study, we found that POMS patients never exposed to DMTs during the observation period had the highest risk of experiencing a first CDW or reaching the EDSS 4.0. Despite the small sample, consistent findings also resulted from sensitivity analyses. Furthermore, also in this cohort, a prolonged DMT exposure was associated with a stepwise risk reduction. Further studies are necessary to assess the safety profile of a prolonged DMT exposure, to establish the role of DMT in cognitive impairment in this subset of patients and to evaluate the effectiveness of an escalation versus induction approach.

In the LOMS cohort, DMTs did not significantly influence the time to the first 12-month CDW, but we found that they significantly reduced the risk of reaching EDSS 4.0. RCTs of DMTs for RRMS were not designed to assess efficacy in ageing patients. Pivotal clinical trials specifically excluded individuals aged $>50$ years [glatiramer acetate (Johnson et al., 1995), IFN 11 b (Paty and Li, 1993), natalizumab (Polman et al., 2006), alemtuzumab (Panitch et al., 2008)] and aged $>55$ years [IFN $\beta 1$ a (Jacobs et al., 2000), dimethyl fumarate (Gold et al., 2012), fingolimod (Kappos et al., 2010), teriflunomide (O'Connor et al., 2011), rituximab (Hauser et al., 2008), ocrelizumab (Hauser et al., 2017) and ozanimod (Comi et al., 2019)]. Moreover, a recent metaanalysis of 38 RCTs found that, after age 53 , there is no predicted benefit of immunomodulatory therapy (Weideman et al., 2017). This model, however, was based on the abovecited clinical trials, so that the analyses were underpowered in patients aged $>50$ years. Furthermore, the model was based on patients' mean outcomes, whereas the clinical choice has to deal with individual patient's outcome. It is, therefore, reasonable to hypothesize that among LOMS patients there can also be responders to DMTs. Indeed, the post hoc analyses of the CLARITY trial (cladribine tablets treating multiple sclerosis orally), where the upper age limit was 65 years (Giovannoni et al., 2010), revealed that the medication was similarly effective in younger and older patients with RRMS using a cut-off of 40 years of age (Giovannoni et al., 2011; Rammohan et al., 2012). Similarly, in the CONCERTO trial on laquinimod, although the primary outcome was negative, the authors were able to find a subgroup of patients that responded to the drug, represented mainly by older females (Bovis et al., 2019). 
Overall, our findings confirmed previous works (Shirani et al., 2015; Guillemin et al., 2017, Weideman et al., 2017; Vaughn et al., 2019) and age-based subgroup analyses of RCTs (Devonshire et al., 2012; Miller et al., 2012) that suggested the existence of different impactful periods of intervention: higher impact in POMS and AOMS and progressively lower, although still detectable, impact in LOMS. At the same time, our results emphasize the importance of further research to clarify the existence of a hypothetical 'upper limit' of age for the response to DMTs; moreover, to characterize good responders among LOMS, in which benefits may overwhelm treatment-related risks. Long-term safety studies are warranted in this more vulnerable population.

Our study also highlighted the prognostic role of relapses and gender.

As expected, the mean number of relapses was higher in POMS than in AOMS and LOMS. Natural history studies in RRMS (Tremlett et al., 2009; Scalfari et al., 2010) found that only early relapses can significantly impact time to EDSS 6.0 and secondary progression. It has also been reported that the effect of relapses is modulated by age, as the impact seems to be higher in younger $(<25$ years at onset) than in older ( $>35$ years) patients (Tremlett et al., 2009). In our three cohorts, the total number of relapses predicted a higher risk of mid-term disability progression. Our study, however, was not specifically designed to explore the effect of relapses on disability, and we did not stratify early versus late relapses. Despite these limitations, we found that a higher number of relapses was associated with a greater risk of disability, also in the LOMS cohort. We can therefore hypothesize that, although neurodegenerative mechanisms may be prevalent in the later stages of the disease, the inflammatory process can persist and impact disability accrual also in older patients.

As for gender, our female:male ratio of 2.00 in LOMS was more elevated compared to previous studies (Polliack et al., 2001; Tremlett and Devonshire, 2006) that did not control for phenotype at onset, while it was similar to that reported in another study (Bove et al., 2012) considering phenotype at onset. Our study suggested that female sex can exert a protective role only in the LOMS cohort. This result was confirmed in sensitivity analyses. Previous studies assessing the effect of gender on disability worsening did not control for progressive compared with relapsing onset (Polliack et al., 2001; Tremlett and Devonshire, 2006). The only study that specifically investigated the effect of gender in RRLOMS found that time to EDSS 6.0 was delayed in AOMS female but not in LOMS female in a large cohort (Bove et al., 2012). Different reasons can explain our conflicting results. First, we assessed the time to EDSS 4.0 after adjusting for baseline EDSS, relapses and treatment exposure. Second, the authors in the cited study did not explicitly report the number of events they registered. Finally, it was a monocentric study, and the presence of referral bias cannot be ruled out. The impact, if any, of menopause on multiple sclerosis course is controversial with some authors reporting no differences in disability progression before and after menopause (Ladeira et al., 2019), while others suggest a possible worsening of multiple sclerosis disability (Bove et al., 2016). At low levels, oestrogens seem to worsen the course of multiple sclerosis, while at higher levels they seem to have anti-inflammatory properties that are under investigation (Collongues et al., 2018). As neither the age at menopause was systematically recorded in Italian multiple sclerosis registry nor the proportion of LOMS female under hormone replacement therapy, we are not able to assess the effect that menopause had in RR-LOMS females. Further investigations are warranted to shed some light in this field.

In interpreting our results, we have to take into account a few study limitations. As we included patients with a first evaluation within 3 years from symptom onset, immortal time bias can represent a concern. Indeed, we were not able to perform a reliable sensitivity analysis because of the limited sample of patients with a first visit within 1 year from disease onset. Furthermore, we included patients diagnosed with different diagnostic criteria, which may lead to a phenomenon known as the Will Rogers effect (Kalincik and Butzkueven, 2016; Trojano et al., 2017). We tried to mitigate the inclusion of 'historical patients' by running sensitivity analyses of patients recorded in the registry after 2000 . However, we were not able to further stratify patients according to their referring centre or region due to the under-representation of POMS and LOMS cohorts. Our patients were mostly treated with moderately effective DMTs, so that results cannot be directly extrapolated to patients treated with highly effective DMTs. Finally, due to the observational nature of the study, the presence of unknown confounders cannot be ruled out.

In conclusion, our real-world data add to available evidence on the critical role of age in the evaluation of the benefit-risk balance and provide some cues to treatment decision-making in younger and older patient populations under-represented in clinical trials.

\section{Funding}

The Italian iMed-Web database has received financial support by annual research grants from the Italian University and Research Ministry (MIUR) (COFIN 2009-2014 M.T.) and from Merck Serono, Novartis Pharma and Biogen. The funders had no role in study design, data collection and analysis, decision to publish, or preparation of the manuscript.

\section{Competing interests}

M.P.A. served on scientific advisory boards for and has received speaker honoraria and research support from Biogen Idec, Merck Serono, Bayer Schering Pharma, and Sanofi Aventis, and serves on the editorial board of BMC Neurology. E.P. served on a scientific advisory board for Biogen and Merck Serono. He received honoraria from 
Biogen, Merck Serono, Teva, Genzyme. L.P. received research support from Novartis, Biogen and speakers honoraria from Teva. L.R. received research support from Novartis. G.C. has received consulting fees from Novartis, Teva Pharmaceutical Industries Ltd, Sanofi-Aventis, Dompè, Bayer-Schering and Serono Symposia International Foundation, and trial grant support from Novartis, Teva Pharmaceutical Ind, Ltd, Sanofi-Aventis, Receptors, Biogen Idec, Genentech-Roche, Merck, Biogen Dompe, and Bayer Schering. F.P. received personal compensation for advisory board and speaking activities from Almirall, Biogen, Cilgane, Merck, Novartis, Roche, Sanofi and Teva. D.L.G. serves on scientific advisory boards and received honoraria and travel grants from Biogen, Merck Serono, Novartis and Roche. V.B.M. public speaking on consultancy from Merck, Novartis, Biogen, Genzyme, Teva and Alrmirall. E.C. received research grants and honoraria as a speaker and member of advisory boards by: Almirall, Bayer, Biogen Idec, Merck Serono, Novartis, Sanofi Genzyme, Teva, Roche. C.P. has served on scientific advisory boards for Actelion, Almirall Biogen, Genzyme, Roche, Merck, Novartis, and has received consulting and/or speaking fees, research support and travel grants from, Almirall, Biogen, Merck, Genzyme, La Roche, Novartis. P.S. has received speaker and advisory boards honoraria and travel grants from TEVA, Merck Serono, Sanofi-Genzyme, Novartis, and Biogen. B.R. received funding for congress/travel/accommodation expenses for scientific meetings and honoraria for speaking from Almirall, Bayer Schering, Biogen, Merck Serono, Novartis, Sanofi-Genzyme, Teva; he served on scientific Advisory Boards for Almirall, Biogen, Merck Serono, Novartis, Sanofi-Genzyme, Teva; he received research grants for his Department by Biogen, Merck Serono, SanofiGenzyme, Teva. G.S. received grants and honoraria by Biogen-Dompè, Sanofi-Aventis, Novartis, Teva, MerckSerono, Almirall, and Roche. I.M. has received research grants from NIH, NMSS, FISM, Teva Neuroscience and honoraria from Genzyme, Roche and Merck. E.M. has received research grants from Roche, Merck, Biogen, Sanofi, Novartis. S.G. has received speaker fees or travel expenses for attending meetings from Biogen, Merck- Serono, Teva Almirall, Sanofi-Aventis, Novartis, Genzyme. A.G. has served on scientific advisory boards for Merck Serono, Biogen Idec and Teva Pharmaceutical Industries Ltd; has received speaker honoraria from Merck Serono, Biogen ldec, Bayer Schering Pharma, Novartis, and Serono Symposium International; served as a consultant for Novartis; and receives research support from SanofiAventis, Biogen Idec and Merck Serono. M.Z. received honoraria for participation in advisory boards or travel grants from Biogen Idec, Sanofi Genzyme, Merck Serono, Novartis, and funding to his institution from Novartis. M.S. has received consulting fees and/or honoraria for speaking and/or research grants from Biogen, Genzyme, Merck Serono, Novartis, Roche, and Teva. G.L. received research grants and honoraria as a speaker and member of advisory boards by: Bayer, Biogen Idec, Merck Serono, Novartis, Sanofi Genzyme, Teva,
Almirall, Allergan, Merz, Ipsen, Roche. C.F. received personal compensation from Merck Serono, Biogen, and TEVA for public speaking and advisory boards. R.T. received funding for travel or speaker honoraria from Alfa Wasserman, Bayer, Biogen, CLS Bering, Merck Serono, Novartis, SanofiAventis, Roche, and TEVA. F.G. reported receiving personal fees from Biogen, Sanofi, Roche, and Merck Serono: grant support from Biogen and Sanofi. M.V. received funding for travel or speaker honoraria from Alfa Biogen, Merck Serono, Novartis, Roche and TEVA. G.D.B. received speaker honoraria and travel grants from Teva, Sanofi Genzyme, Biogen Serono and Novartis. U.A. reports a grant from Biogen (no disclosures relevant to this work). P.I. has served on scientific advisory boards for Biogen Idec, Bayer Teva, Roche, Merck Serono, Novartis and Genzyme and has received funding. M.T. for travel and/or Speaker honoraria from Sanofi Aventis, Genzyme, Biogen Idec, Teva, Merck, Serono and Novartis reported receiving speaker honoraria and research grants to her institution from and serving on advisory boards of Biogen, Merck Serono, and Novartis. All other authors report no competing interests related to this work.

\section{Supplementary material}

Supplementary material is available at Brain online.

\section{References}

Alroughani R, Boyko A. Pediatric multiple sclerosis: a review. BMC Neurol 2018; 18: 27.

Amato MP, Goretti B, Ghezzi A, Hakiki B, Niccolai C, Lori S, et al. Neuropsychological features in childhood and juvenile multiple sclerosis: five-year follow-up. Neurology 2014; 83: 1432-8.

Bove R, Healy BC, Musallam A, Glanz BI, De Jager PL, Chitnis T.Exploration of changes in disability after menopause in a longitudinal multiple sclerosis cohort. Mult Scler 2016; 22: 935-43.

Bove RM, Healy B, Augustine A, Musallam A, Gholipour T, Chitnis T. Effect of gender on late-onset multiple sclerosis. Mult Scler J 2012; 18: 1472-9.

Bovis F, Carmisciano L, Signori A, Pardini M, Steinerman JR, Li T, et al. Defining responders to therapies by a statistical modeling approach applied to randomized clinical trial data. BMC Med 2019; 17: 10 .

Chitnis T, Tenembaum S, Banwell B, Krupp L, Pohl D, Rostasy K, et al. Consensus statement: evaluation of new and existing therapeutics for pediatric multiple sclerosis. Mult Scler J 2012; 18: 116-27.

Collongues N, Patte-Mensah C, De Seze J, Mensah-Nyagan AG, Derfuss T. Testosterone and estrogen in multiple sclerosis: from pathophysiology to therapeutics. Expert Rev Neurother 2018; 18: 515-22.

Comi G, Kappos L, Selmaj KW, Bar-Or A, Arnold DL, Steinman L, et al. Safety and efficacy of ozanimod versus interferon beta-1a in relapsing multiple sclerosis (SUNBEAM): a multicentre, randomised, minimum 12-month, phase 3 trial. Lancet Neurol 2019; 18: 1009-20.

Confavreux C, Vukusic S. Natural history of multiple sclerosis: a unifying concept. Brain 2006a; 129: 606-16.

Confavreux C, Vukusic S. Age at disability milestones in multiple sclerosis. Brain 2006b; 129: 595-605. 
Cree BAC, Gourraud PA, Oksenberg JR, Bevan C, Crabtree-Hartman E, Gelfand JM, et al. Long-term evolution of multiple sclerosis disability in the treatment era. Ann Neurol 2016; 80: 499-510.

Devonshire V, Havrdova E, Radue EW, O'Connor P, Zhang-Auberson L, Agoropoulou C, et al. Relapse and disability outcomes in patients with multiple sclerosis treated with fingolimod: subgroup analyses of the double-blind, randomised, placebo-controlled FREEDOMS study. Lancet Neurol 2012; 11: 420-8.

Finlayson M. Concerns about the future among older adults with multiple sclerosis. Am J Occup Ther 2004; 58: 54-63.

Ghezzi A, Amato MP, Makhani N, Shreiner T, Gärtner J, Tenembaum S. Pediatric multiple sclerosis: conventional first-line treatment and general management. Neurology 2016; 87: S97-102.

Giovannoni G, Comi G, Cook S, Rammohan K, Rieckmann P, Sørensen PS, et al. A placebo-controlled trial of oral cladribine for relapsing multiple sclerosis. N Engl J Med 2010; 362: 416-26.

Giovannoni G, Cook S, Rammohan K, Rieckmann P, Sørensen PS, Vermersch P, et al. Sustained disease-activity-free status in patients with relapsing-remitting multiple sclerosis treated with cladribine tablets in the CLARITY study: a post-hoc and subgroup analysis. Lancet Neurol 2011; 10: 329-37.

Gold R, Kappos L, Arnold DL, Bar-Or A, Giovannoni G, Selmaj K, et al. Placebo-controlled phase 3 study of oral BG-12 for relapsing multiple sclerosis. N Engl J Med 2012; 367: 1098-107.

Guillemin F, Baumann C, Epstein J, Kerschen P, Garot T, Mathey G, et al. Older age at multiple sclerosis onset is an independent factor of poor prognosis: a population-based cohort study. Neuroepidemiology 2017; 48: 179-87.

Hauser SL, Bar-Or A, Comi G, Giovannoni G, Hartung HP, Hemmer $\mathrm{B}$, et al. Ocrelizumab versus interferon beta-1a in relapsing multiple sclerosis. N Engl J Med 2017; 376: 221-34.

Hauser SL, Waubant E, Arnold DL, Vollmer T, Antel J, Fox RJ, et al. B-cell depletion with rituximab in relapsing-remitting multiple sclerosis. N Engl J Med 2008; 358: 676-88.

Iaffaldano P, Simone M, Lucisano G, Ghezzi A, Coniglio G, Brescia Morra V, et al. Prognostic indicators in pediatric clinically isolated syndrome. Ann Neurol 2017; 81: 729-39.

Jacobs LD, Beck RW, Simon JH, Kinkel RP, Brownscheidle CM, Murray TJ, et al. Intramuscular interferon beta-1a therapy initiated during a first demyelinating event in multiple sclerosis. $\mathrm{N}$ Engl J Med 2000; 343: 898-904.

Johnson KP, Brooks BR, Cohen JA, Ford CC, Goldstein J, Lisak RP, et al. Copolymer 1 reduces relapse rate and improves disability in relapsing-remitting multiple sclerosis: results of a phase III multicenter, double-blind placebo-controlled trial. Neurology 1995; 45: 1268-76.

Jokubaitis VG, Spelman T, Kalincik T, Izquierdo G, Grand'Maison F, Duquette P, et al. Predictors of disability worsening in clinically isolated syndrome. Ann Clin Transl Neurol 2015; 2: 479-91.

Jokubaitis VG, Spelman T, Kalincik T, Lorscheider J, Havrdova E, Horakova D, et al. Predictors of long-term disability accrual in relapse-onset multiple sclerosis. Ann Neurol 2016; 80: 89-100.

Kalincik T, Butzkueven H. Observational data: understanding the real MS world. Mult Scler 2016; 22: 1642-8.

Kappos L, Freedman MS, Polman CH, Edan G, Hartung HP, Miller $\mathrm{DH}$, et al. Long-term effect of early treatment with interferon beta$1 \mathrm{~b}$ after a first clinical event suggestive of multiple sclerosis: 5-year active treatment extension of the phase 3 BENEFIT trial. Lancet Neurol 2009; 8: 987-97.

Kappos L, Radue EW, O’Connor P, Polman C, Hohlfeld R, Calabresi $\mathrm{P}$, et al. A placebo-controlled trial of oral fingolimod in relapsing multiple sclerosis. N Engl J Med 2010; 362: 387-401.

Kremenchutzky M, Rice GPA, Baskerville J, Wingerchuk DM, Ebers GC. The natural history of multiple sclerosis: a geographically based study 9: observations on the progressive phase of the disease. Brain 2006; 129: 584-94.

Ladeira F, Salavisa M, Caetano A, Barbosa R, Sá F, Correia AS. The influence of menopause in multiple sclerosis course: a longitudinal cohort study. Eur Neurol 2019; 80: 223-7.
Miller AE, O'Connor P, Wolinsky JS, Confavreux C, Kappos L, Olsson TP, et al. Pre-specified subgroup analyses of a placebo-controlled phase III trial (TEMSO) of oral teriflunomide in relapsing multiple sclerosis. Mult Scler J 2012; 18: 1625-32.

Musella A, Gentile A, Rizzo FR, Vito FD, Fresegna D, Bullitta S, et al. Interplay between age and neuroinflammation in multiple sclerosis: effects on motor and cognitive functions. Front Aging Neurosci 2018; 10: 1-13.

O'Connor P, Wolinsky JS, Confavreux C, Comi G, Kappos L, Olsson $\mathrm{TP}$, et al. Randomized trial of oral teriflunomide for relapsing multiple sclerosis. N Engl J Med 2011; 365: 1293-303.

Panitch H, Anaissie E, Cines D, DeGroot L, Dorsey F, Phillips T, et al. Alemtuzumab vs. interferon beta-1a in early multiple sclerosis. $\mathrm{N}$ Engl J Med 2008; 359: 1786-801.

Paty DW, Li D. Interferon beta-1b is effective in relapsing-remitting multiple sclerosis: II. MRI analysis results of a multicenter, randomized, double-blind, placebo-controlled trial. Neurology 1993; 43: 662-7.

Polliack ML, Barak Y, Achiron A. Late-onset multiple sclerosis. J Am Geriatr Soc 2001; 49: 168-71.

Polman CH, O’Connor PW, Havrdova E, Hutchinson M, Kappos L, Miller DH, et al. A randomized, placebo-controlled trial of natalizumab for relapsing multiple sclerosis. N Engl J Med 2006; 354: 899-910.

Rammohan K, Giovannoni G, Comi G, Cook S, Rieckmann P, Sørensen PS, et al. Cladribine tablets for relapsing-remitting multiple sclerosis: efficacy across patient subgroups from the phase III CLARITY study. Mult Scler Relat Disord 2012; 1: 49-54.

Renoux C, Vukusic S, Mikaeloff Y, Edan G, Clanet M, Dubois B, et al. Natural history of multiple sclerosis with childhood onset. N Engl J Med 2007; 356: 2603-13.

Rotstein D, Montalban X.Reaching an evidence-based prognosis for personalized treatment of multiple sclerosis. Nat Rev Neurol 2019; 15: 287-300.

Scalfari A, Neuhaus A, Daumer M, Deluca GC, Muraro PA, Ebers GC. Early relapses, onset of progression, and late outcome in multiple sclerosis. JAMA Neurol 2013; 70: 214-22.

Scalfari A, Neuhaus A, Degenhardt A, Rice GP, Muraro PA, Daumer $\mathrm{M}$, et al. The natural history of multiple sclerosis, a geographically based study 10: relapses and long-term disability. Brain 2010; 133: 1914-29.

Schweitzer F, Laurent S, Fink GR, Barnett MH, Reddel S, Hartung $\mathrm{HP}$, et al. Age and the risks of high-efficacy disease modifying drugs in multiple sclerosis. Curr Opin Neurol 2019; 32: 305-12.

Shirani A, Zhao Y, Petkau J, Gustafson P, Karim ME, Evans C, et al. Multiple sclerosis in older adults: the clinical profile and impact of interferon beta treatment. Biomed Res Int 2015; 2015: 451912.

Tremlett H, Devonshire V. Is late-onset multiple sclerosis associated with a worse outcome? Neurology 2006; 67: 954-9.

Tremlett H, Paty D, Devonshire V. Disability progression in multiple sclerosis is slower than previously reported. Neurology 2006; 66: 172-7.

Tremlett H, Yousefi M, Devonshire V, Rieckmann P, Zhao Y. Impact of multiple sclerosis relapses on progression diminishes with time. Neurology 2009; 73: 1616-23.

Trojano M, Bergamaschi R, Amato MP, Comi G, Ghezzi A, Lepore V, et al. The Italian multiple sclerosis register. Neurol Sci 2019; 40: 155-65.

Trojano M, Butzkueven H, Kappos L, Wiendl H, Spelman T, Pellegrini F, et al. Natalizumab treatment shows low cumulative probabilities of confirmed disability worsening to EDSS milestones in the long-term setting. Mult Scler Relat Disord 2018; 24: 11-9.

Trojano M, Pellegrini F, Paolicelli D, Fuiani A, Zimatore GB, Tortorella $\mathrm{C}$, et al. Real-life impact of early interferon $\beta$ therapy in relapsing multiple sclerosis. Ann Neurol 2009; 66: 513-20.

Trojano M, Tintore M, Montalban X, Hillert J, Kalincik T, Iaffaldano $\mathrm{P}$, et al. Treatment decisions in multiple sclerosis-insights from realworld observational studies. Nat Rev Neurol 2017; 13: 105-18. 
Tutuncu M, Tang J, Zeid NA, Kale N, Crusan DJ, Atkinson EJ, et al. Onset of progressive phase is an age-dependent clinical milestone in multiple sclerosis. Mult Scler J 2013; 19: 188-98.

Uitdehaag B, Constantinescu C, Cornelisse P, Jeffery D, Kappos L, Jo D, et al. Impact of exposure to interferon beta-1a on outcomes in patients with relapsing-remitting multiple sclerosis: exploratory analyses from the PRISMS long-term follow-up study. Ther Adv Neurol Disord 2011; 4: 3-14.

Uitdehaag B, Kappos L, Verdun E, Gardner J. Continuous versus noncontinuous subcutaneous interferon $\beta-1 \mathrm{a}$ treatment in relapsingremitting multiple sclerosis: long-term data from the PRISMS study. Ther Adv Neurol Disord 2009; 256: S122-3.
Vaughn CB, Jakimovski D, Kavak KS, Ramanathan M, Benedict RHB, Zivadinov R, et al. Epidemiology and treatment of multiple sclerosis in elderly populations. Nat Rev Neurol 2019; 15: 329-42.

Waubant E, Banwell B, Wassmer E, Sormani MP, Amato MP, Hintzen $\mathrm{R}$, et al. Clinical trials of disease-modifying agents in pediatric MS: opportunities, challenges, and recommendations from the IPMSSG. Neurology 2019; 92: E2538-49.

Weideman AM, Tapia-Maltos MA, Johnson K, Greenwood M, Bielekova B. Meta-analysis of the age-dependent efficacy of multiple sclerosis treatments. Front Neurol 2017; 8: 1-12. 